\title{
Giant endocarditis vegetation on a pace-maker lead
}

\author{
Emanuele Durante-Mangoni · Gianantonio Nappi
}

Received: 7 November 2009/Accepted: 18 November 2009/Published online: 3 December 2009 (C) SIMI 2009

A 19 year-old man presented with a 2-month history of fever, cough and hemoptysis that were unresponsive to broad spectrum antibiotics. He had been implanted with an epicardial pace-maker at the age of 5 for complete heart block. One year before presentation, he experienced rupture of the epicardial implant following a car accident, and underwent emergency placement of an endocardial pacemaker. He also underwent orthopedic and abdominal surgery for multiple bone fractures and mesenteric artery aneurysm, with a resultant long-lasting intensive care unit stay.

On admission to our hospital, computed tomography of the chest showed bilateral pulmonary inflammatory infiltrates with multiple pulmonary artery emboli. Trans-thoracic echocardiography showed a massive, mobile structure adhering to the pace-maker lead and severe pulmonary hypertension. Multiple blood cultures grew Candida albicans. The patient remained highly febrile and septic despite full dose combination antifungal treatment with caspofungin and fluconazole.

E. Durante-Mangoni · G. Nappi

Department of Cardiothoracic Sciences,

Second University of Naples,

Via Leonardo Bianchi snc, 80131 Naples, Italy

E. Durante-Mangoni · G. Nappi

Department of Cardiovascular Surgery and Transplants,

Monaldi Hospital, Via Leonardo Bianchi snc,

80131 Naples, Italy

\section{E. Durante-Mangoni $(\square)$}

Cattedra di Medicina Interna SUN, UOC Medicina

Infettivologica e dei Trapianti, AORN 'V. Monaldi',

Via Bianchi snc, 80131 Naples, Italy

e-mail: emanuele.durante@unina2.it
On the fourth hospital day, due to persistent sepsis and high embolic risk, he underwent open-heart removal of a giant $5.5 \times 2.0 \mathrm{~cm}$ vegetation adhering to the pace-maker lead (Fig. 1). Excision was done through the right atrium on total cardio-pulmonary bypass but with a beating heart. A pace-maker was again placed through an epicardial lead. Although a small vegetation was still detectable at echocardiography on the ventricular side of the tricuspid valve, the patient recovered fully on combination antifungal treatment, and was discharged afebrile 3 weeks later.

The screening for thrombophilia showed that the patient had hyper-homocysteinemia $(20 \mathrm{mmol} / \mathrm{l})$ due to homozygous C677T polymorphism of the methylene-tetra-hydropholate reductase gene. Antifungal treatment was given for a total of 8 weeks while oral anticoagulation with warfarin was continued indefinitely. Supplementation with folates and group B vitamins was also given. The patient has remained stable without any sign of recurrence 3 months after discharge.

Large endocardial vegetations are a frequent finding in cases of fungal endocarditis [1]. However, a vegetation length greater than $3 \mathrm{~cm}$ is exceedingly rare. In our case, the co-existence of an inherited thrombophilic state may have contributed to the exceptional growth of the vegetation [2]. The current guidelines suggest early cardiac surgical intervention in cases of fungal endocarditis, especially if a prosthetic intracardiac device is involved [3]. Nonetheless, the availability of novel antifungal agents with improved fungicidal activity and a high penetration coefficient within fungal biofilms will likely improve the outcome of these patients [1]. Acquisition of blood-stream fungal infections is often health care related, and its incidence is growing [4]. The described case further highlights the importance of adhering to asepsis protocols while 


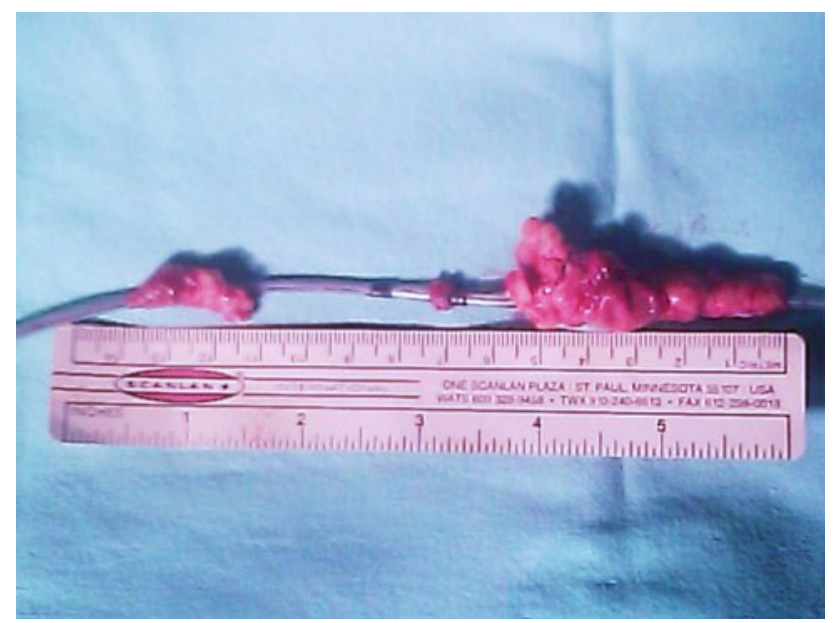

Fig. 1 Three endocarditis vegetations were found on the explanted pace-maker lead. The larger one, measuring $5.5 \mathrm{~cm}$, had nearly filled the right ventricle and showed systolic movement into the pulmonary artery root. A smaller, $2.5 \mathrm{~cm}$ long, vegetation was found on the atrial section of the lead. A further, minor, vegetation was also present where the pace-maker lead crossed the tricuspid valve

caring for subjects with endovascular devices in order to minimize the risk of infection.

Conflict of interest statement The authors declare that they have no conflict of interest related to the publication of this manuscript.

\section{References}

1. Falcone M, Barzaghi N, Carosi G, Grossi P, Minoli L, Ravasio V, Rizzi M, Suter F, Utili R, Viscoli C, Venditti M, (2009) Italian Study on Endocarditis. Candida infective endocarditis: report of 15 cases from a prospective multicenter study. Medicine (Baltimore) $88: 160-168$

2. Durante-Mangoni E, Brugnone R, Iossa D, Molaro R, Crispi F, Albisinni R, Caprioli V, Utili R (2009) Infective endocarditis on cardiac rhythm management devices: preliminary study on the possible pathogenic role of inherited thrombophilias. Int $\mathrm{J}$ Antimicrob Agents 33:S15

3. Task Force on the Prevention, Diagnosis, and Treatment of Infective Endocarditis of the European Society of Cardiology, European Society of Clinical Microbiology and Infectious Diseases, International Society of Chemotherapy for Infection and Cancer, Habib G, Hoen B, Tornos P et al (2009) Guidelines on the prevention, diagnosis, and treatment of infective endocarditis (new version 2009): The Task Force on the Prevention, Diagnosis, and Treatment of Infective Endocarditis of the European Society of Cardiology (ESC). Eur Heart J 30:2369-2413

4. Murdoch DR, Corey GR, Hoen B et al (2009) Clinical presentation, etiology, and outcome of infective endocarditis in the $21 \mathrm{st}$ Century. The International Collaboration on Endocarditis-Prospective Cohort Study. Arch Intern Med 169:463-473 